\title{
Changes in developmental state: demolish the old to construct the new
}

\author{
Warren P. Voth and David J. Stillman ${ }^{1}$ \\ Department of Pathology, University of Utah, Salt Lake City, Utah 84132, USA
}

Development, along with other cellular changes of state, can be conceived as the sequential adoption of stable, epigenetically determined patterns of gene expression. The article by Laney and Hochstrasser (2003) in this issue is the most recent to exploit the Saccharomyces cerevisiae mating-type system to characterize molecular mechanisms that act when cells change gene expression and cellular state. They examine the relationship between transcriptional regulators of mating type and components of the ubiquitin-mediated protein degradation pathway, and demonstrate that proteolysis is required not only to end one program of gene expression, but also to prevent an erroneous subsequent transcriptional state.

Laney and Hochstrasser (2003) conclude that ubiquitin-mediated proteolysis of the Mat $\alpha 2$ transcriptional repressor is fundamentally required for the developmental transition that is the yeast mating-type switch. Their work is dedicated to the memory of Ira Herskowitz, and this is a fitting tribute because he did so much to further our understanding of mating-type switching and the regulation of mating type by regulatory factors. Herskowitz coined the term "master regulatory" gene (Herskowitz 1985) and applied it to the regulators of mating type; this term has also been adopted for critical transcriptional regulators in metazoan development (Weintraub et al. 1989; Baker 2001).

\section{Regulation of mating type}

Saccharomyces lives vegetatively in either the haploid or the diploid state. Haploids are found in two mating types, MATa or MAT $\alpha$, which mate with each other to form the preferred diploid cell type. In 1981, Herskowitz and colleagues proposed that the MATa and MAT $\alpha$ loci encode regulatory molecules that control the expression of other genes that determine the phenotype of cells (Strathern et al. 1981). They proposed that in $\alpha$ cells, Mat $\alpha 1$ functioned as an activator, whereas Mat $\alpha 2$ functioned as a repressor, and that in diploids, Matal and Mat $\alpha 2$ worked together as the a1/ $\alpha 2$ repressor (Fig. 1). This $\alpha 1 / \alpha 2$ hypothesis was a remarkably prescient prediction, as it was based solely on genetic analysis. Sub-

${ }^{1}$ Corresponding author.

E-MAIL david.stillman@path.utah.edu; FAX (801) 581-3607.

Article and publication are at http://www.genesdev.org/cgi/doi/10.1101/ gad.1142103. sequent cloning and sequencing of the $M A T$ genes showed that they encoded homeodomain transcription factors, and that these proteins recognized sites in the promoters of the relevant target genes.

Phenotypically, a and $\alpha$ cells differ from each other only in the expression of genes controlling the production and sensing of mating pheromones. a-specific genes are expressed only in a cells, but these genes are repressed in $\alpha$ cells by $\alpha 2$, along with the abundant and pleiotropic regulator $\mathrm{Mcm} 1$ and the Ssn6 and Tup1 corepressors (Fig. 1; for review, see Sprague 1990). $\alpha$-specific genes are expressed only in $\alpha$ cells, because they are activated by $\alpha 1$, also in concert with Mcm1. In diploid cells, haploid-specific genes, required for mating or to block meiosis, are repressed by the heterodimeric a1/ 2 transcription factor, composed of one protein expressed from MATa and one from MAT $\alpha$. a1/ $\alpha 2$ also recruits Mcm1 and Ssn6-Tup1 in order to repress haploid-specific genes in diploid cells. In a cells, a-specific genes are expressed without any mating-type specific factor, as the a cell type is a default ground state. Thus Mata1 has no role in a haploid cells, but functions only in the diploid heterodimeric context.

\section{Mating-type switching}

Genetically, haploid yeast can switch to the opposite mating type by changing the identity of the MAT locus DNA from that encoding the Matal transcription factor to one encoding Mat $\alpha 1$ and Mat $\alpha 2$, or vice versa (Haber 1998). This switching is very efficient, occuring every generation in the larger mother cells arising from the asymmetric mitosis in yeast. This genetic change is a gene-conversion event initiated by the $H O$ endonuclease. (Diploids do not switch because $H O$ is a haploidspecific gene, one of the targets of a1/ 22 diploid repression.) The $H O$ endonuclease cleaves at the transcriptionally active MAT locus, already containing either a- or $\alpha$-transcription factor-encoding information, and this double-stranded break is repaired by gene conversion using one of two transcriptionally silent loci, $H M L \alpha$ or $H M R a$, that contain information for the regulatory genes. Note that the vast majority of laboratory yeast strains are heterothallic, containing a mutation in the HO gene (Meiron et al. 1995), or else it would be impossible to maintain stable haploid strain stocks. However, 


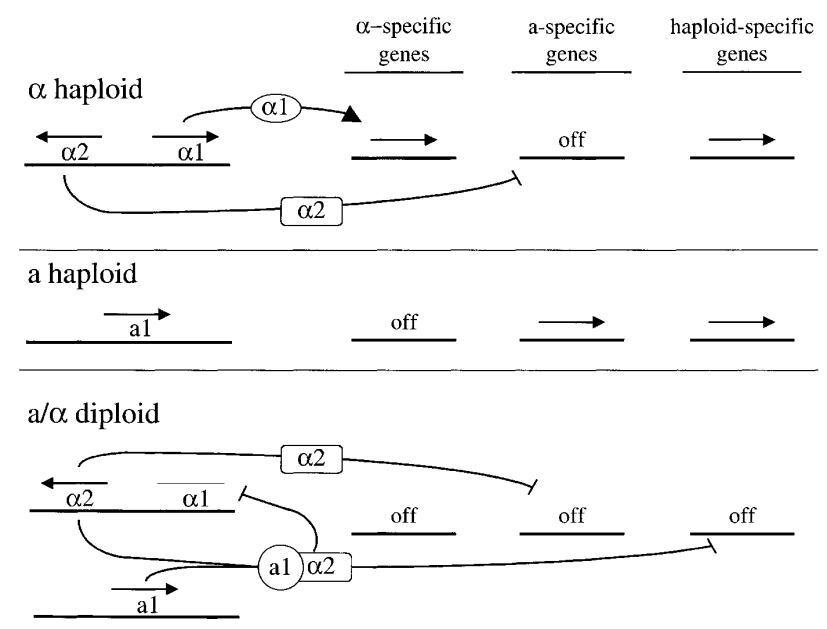

Figure 1. Control of mating type by MAT. In a MAT $\alpha$ haploid cell, the Mat $\alpha 1$ protein activates expression of $\alpha$-specific genes, and the Mat $\alpha 1$ protein represses expression of a-specific genes. Expression of haploid-specific genes does not require any $M A T$ encoded activator. In a MATa haploid cell, the Matal protein is expressed, but it is not required to specify the MATa phenotype, as a-specific genes and haploid-specific genes are expressed by constitutive activators, and the $\alpha$-specific genes are not expressed due to the absence of their activators. In a $/ \alpha$ diploids, the Mata 1 and Mat $\alpha 2$ proteins form the $\mathbf{a} 1 / \alpha 2$ heterodimer that blocks expression of haploid-specific genes. a1 $/ \alpha 2$ also represses expression of $M A T \alpha 1$, and as the Mat $\alpha 1$ protein is not expressed, there is no activator for the $\alpha$-specific genes. Additionally, the Mat $\alpha 2$ protein blocks expression of a-specific genes.

Laney and Hochstrasser (2003) used homothallic strains that switch mating type, allowing sensitive in vivo assays for MAT protein function.

Haploid yeast life history usually consists of rapid homothallic switching of mating type, followed by rapid mating to form a diploid, which is the predominant natural and presumably preferred form. The mechanism of cell-type specification utilizes Mat $\alpha 2$ as a potent repressor in two contexts (Fig. 1). Both cause difficulties for the newly switched MATa cell in preparing to mate and diploidize. Thus, a change in mating cell type must proceed not only by the initiation of a new phenotypic expression program, but also by the inactivation of the determinants of the prior cell type. Herskowitz predicted that many of the components involved in celltype specification, including cell-surface receptors and regulatory proteins such as Mat $\alpha 2$ would, by necessity, be unstable proteins (Herskowitz 1986). In fact, the halflife of Mat $\alpha 2$ is only about 5 min (Hochstrasser and Varshavsky 1990).

\section{Ubiquitin-mediated protein degradation}

Conjugation of ubiquitin to a protein usually leads to formation of a multiubiquitin chain, resulting in degradation of the protein by the proteosome (Hershko and Ciechanover 1998). Protein ubiquitylation occurs via a multienzyme cascade. First, the 76-amino acid ubiquitin polypeptide is activated by covalent attachment to the E1 ubiquitin-activating enzyme, and then transferred to an E2 ubiquitin-conjugating enzyme. Finally, the E2 ubiquitin conjugate and an E3 ubiquitin-protein ligase transfer the ubiquitin to the substrate protein. Most cells have many different E2 and E3 enzymes, which are responsible for the specificity in substrate recognition. The spectrum of specificity is probably enhanced by combinatorial relationships among the E2s and E3s. In Saccharomyces, there are at least three E1 ubiquitin-activating enzymes, 13 E2 ubiquitin-conjugating enzymes (Ubc), and eight E3 ubiquitin-protein ligases (Hochstrasser 1996). Protein degradation-mediated cellular processes include mitotic progression, DNA repair, stress response, signal transduction, metabolic regulation, and epigenetic developmental programming and differentiation. Not all of these processes are dependent on the well-characterized final resting place of many ubiquitylated proteins, the $26 \mathrm{~S}$ proteosome, nor are all protein-degradation processes mediated by ubiquitin.

\section{The unstable Mat $\alpha 2$ protein is ubiquitylated}

It was shown previously that the Mat $\alpha 2$ protein is unstable in vivo, and that it is ubiquitylated prior to destruction (Hochstrasser et al. 1991). Two distinct ubiquitin-addition pathways act on Mat $\alpha 2$; one utilizes the E2s Ubc4 and Ubc5, but the determinants of interaction within Mat $\alpha 2$ are unknown. The second system works through the E2s Ubc6 and Ubc7, and recognizes a signal in Mat $\alpha 2$ termed Deg1, containing an exposed hydrophobic surface of an amphipathic helix at its $\mathrm{N}$ terminus. Using a sensitive genetic selection scheme based on stabilization of the Deg1 domain, the novel E3-encoding DOA10 (degradation of alpha2) gene was isolated as necessary for the turnover of Deg1-containing proteins (Chen et al. 1993). Comfortingly, mutations in ubc6 and $u b c 7$ were also recovered in this screen. Doal0 activity is specific to the second Deg1-dependent pathway.

Now, Laney and Hochstrasser (2003) investigate the relative contributions of these two degradation pathways to Mat $\alpha 2$ turnover. They find that although either the ubc4 or the ubc6 (also doa10) single mutations have minor effects on stabilization of Mat $\alpha 2$, the remaining pathway that is still active elicits near-normal degradation rates. Only in the ubc4 ubc6 (or ubc4 doa10) doublemutant strains is Mat $\alpha 2$ significantly more prevalent, both at the level of bulk Mata2 protein, as well as that fraction bound to the chromatin of repressed genes in vivo. Most importantly, they show that there are phenotypic consequences in these doubly mutant strains, resulting in a defect in diploidization through mating after a mating-type switch. This phenotype is specific to Mat $\alpha 2$ stabilization and not to hyperactivation or stabilization of some other aspect of the switching or mating process.

\section{Mat 2 stabilization prevents mating after a mating-type switch}

To further analyze these phenotypic consequences of Mat $\alpha 2$ stabilization, Laney and Hochstrasser (2003) used 
several assays for mating function. Homothallic strains, with the wild-type $H O$ gene, are competent as haploids to switch mating type in either direction, but in diploids $H O$ is not expressed and switching does not occur (Nasmyth 1993). Homothallic diploids are induced to undergo meiosis, haploid spores are isolated and germinated, and after sufficient growth to generate a colony, their mating ability can be sensitively assessed with a bioassay. Homothallic haploid spores efficiently switch mating type, then mate with closely apposed siblings during outgrowth of the germinated spore, and thus form diploids. This is evidenced by their virtually complete inability to mate with haploid tester cells, as genes required for mating are repressed in diploids by a1/ 2 . (Only cells that can mate with the tester strain form colonies in this assay, because the resulting diploids have complementing auxotrophic nutritional markers.) In contrast, ubc4 ubc6 or ubc4 doa10 double-mutant strains form colonies at high efficiency in this mating assay, because they mate with the tester strain, indicating their previous failure to efficiently switch mating type phenotypically, and thus mate with siblings. Thus, the $u b c / d o a$ strains containing stabilized Mat $\alpha 2$ are defective in diploidization, which requires a rapid change in cellular phenotype.

A second mating assay used to investigate the phenotypic effect of Mat $\alpha 2$ stabilization is the single-cell bioassay known as the $\alpha$-factor pheromone confrontation assay (Hicks and Herskowitz 1976). Homothallic diploids are sporulated and germinated in the presence of $\alpha$-factor. One half of the spores will be of the MATa mating type, and these cells will respond to $\alpha$-factor by arresting in G1 of the cell cycle and forming a microscopically observable change in cell shape known as shmoos. In this assay, we are interested in what happens to the MAT $\alpha$ spores. MAT $\alpha$ cells are insensitive to $\alpha$-factor, and after germination, they divide into two cells. After each mitosis, in the next G1 phase, one of the two cells will undergo a mating-type switch. The switch always occurs in the mother cell, the larger of the two mitotic progeny (Strathern and Herskowitz 1979). A mating-type switching event in an $\alpha$ cell can be scored, because after this cell divides, it gives rise to two a cells that arrest in response to the $\alpha$-factor and form shmoos. Laney and Hochstrasser (2003) found that the increased Mat $\alpha 2$ stability in the $u b c 4$ ubc6 double-mutant blocks mating-type switching. Importantly, whereas phenotypic switches from $\alpha$ to a mating type are inhibited in the ubc4 ubc6 mutant, the reverse switches from a to $\alpha$ mating type occur normally. Additionally, switching was normal in the ubc4 and ubc6 single mutants.

\section{Mat 2 stabilization does not affect mating-type switching at the DNA level}

Whereas the failure to switch mating-type phenotype in the $u b c 4$ ubc6 mutant is ascribed to Mat 2 hyperstabilization, it is possible that these mutations cause a defect in switching at the genotypic level. Mating-type switching involves cleavage of MAT DNA by the $H O$ endo- nuclease, followed by physical recombination leading to replacement of the sequences at the MAT locus. Expression of the $H O$ gene is subject to a complex network of both positive and negative controls (Nasmyth 1993), and it is possible that the $u b c$ mutations affect $H O$ regulation or some aspect of the gene conversion process. Laney and Hochstrasser (2003) monitored DNA-level recombination at the $M A T$ locus in a bulk assay assessing the structure of MAT DNA by Southern blotting. They also monitored gene conversion at the single-cell level by monitoring transposition of GFP from the silent $H M R a$ locus, the donor for recombination, into the transcriptionally active MAT locus. DNA-level switching was unaltered in the $u b c /$ doa mutant contexts in both assays.

\section{Mat 2 stabilization affects switching in two ways}

The authors investigate the mechanism of the switching defect caused by the long-lived Mat $\alpha 2$ protein. Mat $\alpha 2$ regulates gene expression in two ways, by repressing aspecific genes in $\alpha$ cells, and by repressing haploid-specific genes by the a1/ $\alpha 2$ heterodimer (Fig. 1). Thus, a defect in degradation of Mat $\alpha 2$ could result in two types of misprogramming in the new MATa state that would block mating competence. A continued repression of aspecific genes or an adoption of a pseudo-diploid state due to a1/ $\alpha 2$-based repression of haploid-specific genes.

One a-specific gene is STE2, encoding the $\alpha$-pheromone receptor, whose expression is critically required for mating in MATa cells. To address whether a failure to express STE2 underlies the mating defect in the strains with stabilized Mat $\alpha 2$, STE2 was ectopically expressed from a different promoter and found to provide modest rescue of the phenotypic switching defect. To test the role of haploid-specific repression by inappropriately stabilized Mat $\alpha 2$ present in a1/ 22 heterodimers, the $M A T a 1$ gene was deleted from the transcriptionally silent $H M R a$ locus. As $H M R a$ is the donor for the matingtype transposition, gene conversion will produce a switched cell in which there is no Matal protein. This experiment also allows modest rescue of the ubc4 doa10 switching defect. Importantly, combining both the ectopically expressed STE2 and the MATa1 deletion in the same ubc4 doa10 strain results in essentially wild-type levels of switching. This is an incisive observation, indicating that both types of inappropriate repression by the remaining Mat $\alpha 2$ must be relieved for the new developmental program to be manifested. Thus, in strains with stabilized Mat $\alpha 2$, repression of a-specific genes by Mat $\alpha 2$ and haploid-specific genes by a1/ $\alpha 2$ both contribute to the mating defect.

\section{Ubiquitin-mediated destruction of transcription factors}

The majority of transcription factors are unstable, and for many of these factors, it has been shown that degradation occurs following ubiquitylation (Conaway et al. 2002; Muratani and Tansey 2003). It may be that tran- 
scription factors with an intrinsically short half life are important to allow cells to transition from one developmental state to another, as observed for yeast cells switching mating type. Additionally, recent work suggests that ubiquitylation of transcription factors plays an important positive role in transcriptional activation per se, as there is a correspondence between transcriptional activation domains and degrons, sequences that promote degradation (Salghetti et al. 2000, 2001). The Rsp5/ hPRF1 E3 ubiquitin ligases function as coactivators for the steroid-hormone receptor family of transcription factors (Imhof and McDonnell 1996). Salghetti et al. (2001) showed that a met30 mutation that prevents ubiquitylation of the artificial LexA-VP16 activator results in protein stabilization, but this mutation also eliminates the ability of the protein to function as an activator. Moreover, the Gal4 activator recruits the $19 \mathrm{~S}$ regulatory particle of the proteosome to the promoter (Gonzalez et al. 2002). Taken together, these results have generated suicide models, in which DNA-binding by a transcription factor leads to ubiquitylation and rapid destruction, thus ensuring that the transcription signal is as short lived as the transcription factor (Conaway et al. 2002; Muratani and Tansey 2003).

However, the situation with Mat $\alpha 2$ is different, as this protein functions as a repressor, and thus does not have an activation domain. Further work will be needed to understand the difference between unstable activators and unstable repressors, as both are ubiquitylated before destruction, but ubiquitylation apparently contributes to the activation potential for activators. Nonetheless, it seems likely for both activators and repressors that inherent instability is important for cells to be able to change programs of gene expression during development.

\section{References}

Baker, N.E. 2001. Master regulatory genes; telling them what to do. Bioessays 23: 763-766.

Chen, P., Johnson, P., Sommer, T., Jentsch, S., and Hochstrasser, M. 1993. Multiple ubiquitin-conjugating enzymes participate in the in vivo degradation of the yeast MAT $\alpha 2$ repressor. Cell 74: 357-369.

Conaway, R.C., Brower, C.S., and Conaway, J.W. 2002. Emerging roles of ubiquitin in transcription regulation. Science 296: $1254-1258$.

Gonzalez, F., Delahodde, A., Kodadek, T., and Johnston, S.A. 2002. Recruitment of a $19 \mathrm{~S}$ proteasome subcomplex to an activated promoter. Science 296: 548-550.

Haber, J.E. 1998. Mating-type gene switching in Saccharomyces cerevisiae. Annu. Rev. Genet. 32: 561-599.

Hershko, A. and Ciechanover, A. 1998. The ubiquitin system. Annu. Rev. Biochem. 67: 425-479.

Herskowitz, I. 1985. Master regulatory loci in yeast and $\lambda$. Cold Spring Harb. Symp. Quant. Biol. 50: 565-574.

. 1986. Specialized cell types in yeast: Their use in addressing problems in cell biology. In Yeast cell biology. (ed. J. Hicks), pp. 625-656. Alan R. Liss, Inc., New York.

Hicks, J.B. and Herskowitz, I. 1976. Interconversion of yeast mating types. I. Direct observation of the action of the homothallism (HO) gene. Genetics 83: 245-258.

Hochstrasser, M. 1996. Ubiquitin-dependent protein degrada- tion. Annu. Rev. Genet. 30: 405-439.

Hochstrasser, M. and Varshavsky, A. 1990. In vivo degradation of a transcriptional regulator: The yeast a2 repressor. Cell 61: 697-708.

Hochstrasser, M., Ellison, M.J., Chau, V., and Varshavsky, A. 1991. The short-lived MAT $\alpha 2$ transcriptional regulator is ubiquitinated in vivo. Proc. Natl. Acad. Sci. 88: 4606-4610.

Imhof, M.O. and McDonnell, D.P. 1996. Yeast RSP5 and its human homolog hRPF1 potentiate hormone-dependent activation of transcription by human progesterone and glucocorticoid receptors. Mol. Cell. Biol. 16: 2594-2605.

Laney, J.D. and Hochstrasser, M. 2003. Ubiquitin-dependent degradation of the yeast Mat $\alpha 2$ repressor enables a switch in developmental state. Genes \& Dev. (this issue).

Meiron, H., Nahon, E., and Raveh, D. 1995. Identification of the heterothallic mutation in $\mathrm{HO}$-endonuclease of $\mathrm{S}$. cerevisiae using HO/ho chimeric genes. Curr. Genet. 28: 367-373.

Muratani, M. and Tansey, W.P. 2003. How the ubiquitin-proteasome system controls transcription. Nat. Rev. Mol. Cell. Biol. 4: 192-201.

Nasmyth, K. 1993. Regulating the $H O$ endonuclease in yeast. Curr. Opin. Genet. Dev. 3: 286-294.

Salghetti, S.E., Muratani, M., Wijnen, H., Futcher, B., and Tansey, W.P. 2000. Functional overlap of sequences that activate transcription and signal ubiquitin-mediated proteolysis. Proc. Nat1. Acad. Sci. 97: 3118-3123.

Salghetti, S.E., Caudy, A.A., Chenoweth, J.G., and Tansey, W.P. 2001. Regulation of transcriptional activation domain function by ubiquitin. Science 293: 1651-1653.

Sprague Jr., G.F. 1990. Combinatorial associations of regulatory proteins and the control of cell type in yeast. Adv. Genet. 27: 33-62.

Strathern, J.N. and Herskowitz, I. 1979. Asymmetry and directionality in production of new cell types during clonal growth: The switching pattern of homothallic yeast. Cell 17: 371-381.

Strathern, J., Hicks, J., and Herskowitz, I. 1981. Control of cell type in yeast by the mating type locus. The $\alpha 1-\alpha 2$ hypothesis. J. Mol. Biol. 147: 357-372.

Weintraub, H., Tapscott, S.J., Davis, R.L., Thayer, M.J., Adam, M.A., Lassar, A.B., and Miller, A.D. 1989. Activation of muscle-specific genes in pigment, nerve, fat, liver, and fibroblast cell lines by forced expression of MyoD. Proc. Natl. Acad. Sci. 86: 5434-5438. 


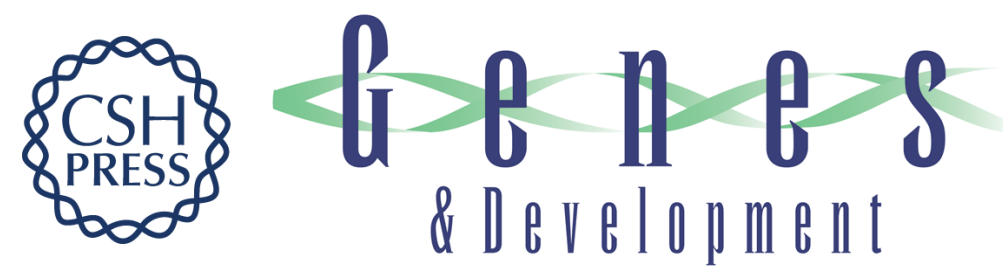

\section{Changes in developmental state: demolish the old to construct the new}

Warren P. Voth and David J. Stillman

Genes Dev. 2003, 17:

Access the most recent version at doi:10.1101/gad.1142103

References This article cites 21 articles, 9 of which can be accessed free at: http://genesdev.cshlp.org/content/17/18/2201.full.html\#ref-list-1

License

Email Alerting

Receive free email alerts when new articles cite this article - sign up in the box at the top Service right corner of the article or click here.

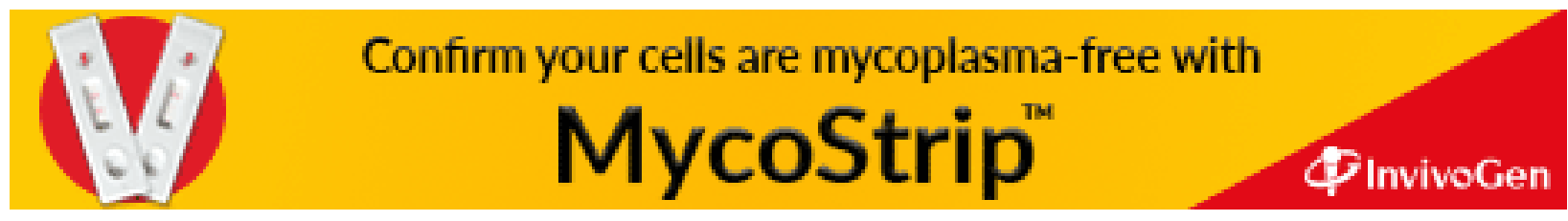

